\title{
Arabidopsis CROWDED NUCLEI (CRWN) proteins are required for nuclear size control and heterochromatin organization
}

\author{
Haiyi Wang ${ }^{1}$, Travis A Dittmer ${ }^{2}$ and Eric J Richards ${ }^{1 *}$
}

\begin{abstract}
Background: Plant nuclei superficially resemble animal and fungal nuclei, but the machinery and processes that underlie nuclear organization in these eukaryotic lineages appear to be evolutionarily distinct. Among the candidates for nuclear architectural elements in plants are coiled-coil proteins in the NMCP (Nuclear Matrix Constituent Protein) family. Using genetic and cytological approaches, we dissect the function of the four NMCP family proteins in Arabidopsis encoded by the CRWN genes, which were originally named LINC (LITTLE NUCLE).
\end{abstract}

Results: CRWN proteins are essential for viability as evidenced by the inability to recover mutants that have disruptions in all four CRWN genes. Mutants deficient in different combinations of the four CRWN paralogs exhibit altered nuclear organization, including reduced nuclear size, aberrant nuclear shape and abnormal spatial organization of constitutive heterochromatin. Our results demonstrate functional diversification among CRWN paralogs; CRWN1 plays the predominant role in control of nuclear size and shape followed by CRWN4. Proper chromocenter organization is most sensitive to the deficiency of CRWN4. The reduction in nuclear volume in crwn mutants in the absence of a commensurate reduction in endoreduplication levels leads to an increase in average nuclear DNA density.

Conclusions: Our findings indicate that CRWN proteins are important architectural components of plant nuclei that play diverse roles in both heterochromatin organization and the control of nuclear morphology.

Keywords: Nuclear organization, Nuclear morphology, Nuclear DNA density, Heterochromatin

\section{Background}

The cellular components and processes that specify nuclear size, shape and internal organization are poorly understood, particularly in flowering plants. Despite similarities at the gross morphological level among all eukaryotic nuclei, such as a double-membrane boundary perforated with nuclear pores, most of the proteins known to affect nuclear structure in animals are not evolutionarily conserved and are consequently difficult to recognize or absent entirely in plant proteomes [1-3]. These observations indicate that the machinery, and perhaps the principles, specifying nuclear organization in flowering plants are distinct from those operating in animals and represent a convergent evolutionary path to a canonical nuclear organization in eukaryotic cells [4].

\footnotetext{
*Correspondence: ejr77@cornell.edu

${ }^{1}$ Boyce Thompson Institute for Plant Research, 533 Tower Road, Ithaca, NY 14853, USA

Full list of author information is available at the end of the article
}

We demonstrated previously $[5,6]$ that two paralogous Arabidopsis coiled-coil proteins, originally named LITTLE NUCLEI 1 and 2 (LINC1 \& 2), play important roles in specifying nuclear shape and size. Supporting this conclusion, Sakamoto and Takagi recently reported that disruption of LINC4, another of the four paralogous genes in this family, leads to reduced nuclear size and loss of elongated nuclear shape in differentiated cells, mirroring the phenotype of linc1 mutants [7]. These proteins are closely related to NMCP1, Nuclear Matrix Constituent Protein 1, originally identified as a protein residing on the periphery of carrot nuclei and a component of the salt-resistant nuclear matrix [8]. Although NMCP1 and related proteins are plant-specific and share no significant amino acid similarity to lamins, their tripartite structure with an extensive central coiled-coil domain and their localization at the nuclear periphery suggest that NMCP1-related plant proteins might be functional analogs of this core component of the animal 
nuclear lamina $[9,10]$. More recent computational analysis [11], however, has suggested that the NMCP class of plant proteins shares more structural similarities to myosins or paramyosins than to lamins.

Here, we extend our reverse genetic analysis to encompass all four members of the Arabidopsis NMCPrelated protein family, which we have renamed CRWN (CROWDED NUCLEI) to avoid confusion with the acronym LINC (LINKER of NUCLEOSKELETON and CYTOSKELETON) that refers to SUN-KASH protein linkages that bridge the inner and outer nuclear membrane [12-16]. Our findings demonstrate that CRWN proteins are essential for viability, and our analyses uncover complex functional diversification among CRWN proteins with regards to their effects on whole-plant morphology, nuclear size, and the spatial organization of constitutive heterochromatin aggregates (chromocenters) in interphase nuclei. We found that CRWN1 plays the most prominent role among CRWN paralogs in controlling nuclear size, while CRWN4 has the most important role in controlling the distribution and number of heterochromatic chromocenters. The reduced nuclear size in crwn mutants is not matched by a commensurate reduction in endopolyploid levels, resulting in increased nuclear DNA densities (mass per unit volume) up to four-fold higher than wild type levels.

\section{Results}

We performed a phylogenetic analysis of Arabidopsis CRWN proteins and their homologues in other species to begin our investigation of the potential diversification within this family. The predicted Arabidopsis proteome contains four closely related CRWN proteins (CRWN1 through 4) that share $30-40 \%$ amino acid identity; no other Arabidopsis proteins with extended regions of significant amino acid identity to CRWN proteins were found. Similar proteins were found in other plant species, but interestingly, no fungi or animal CRWN homologues were identified from searches of protein databases. In addition, CRWN homologues were absent in the predicted proteome of the green algae Chlamydomonas and Volvox.

We constructed a phylogram of CRWN proteins and related plant homologs using a maximum likelihood algorithm (Figure 1 and Additional file 1: Table S1). The tree features two major clades distinct from CRWN homologues in two basal plants, Selaginella moellendorffii and Physcomitrella patens. One clade includes three of the Arabidopsis paralogs, CRWN1, CRWN2 and CRWN3, while CRWN4 belongs to the other clade. Within each clade, the monocot proteins, represented by maize, sorghum and rice, group independently from the dicot proteins. Only two CRWN paralogs exist in these monocots - one CRWN1-like and one CRWN4-like. However, certain dicot species, such as Arabidopsis, poplar, grape, and castor bean, contain multiple copies of CRWN1-like proteins. The dicot CRWN4-like proteins are also distinct from their monocot counterparts in lacking a conserved amino acid motif at the extreme Cterminus (yellow inset in Figure 1 and Additional file 2).

\section{Genetic redundancy in the CRWN family}

The inference that CRWN4 and related proteins are divergent from members of the CRWN1-containing clade was supported by genetic analyses to dissect the functions of the CRWN paralogs. We used Agrobacterium T-DNA insertion alleles to study the effects of inactivating different combinations of CRWN genes [17]. Previously, we demonstrated that the crwn1-1 and crwn2-1 T-DNA alleles severely reduce or eliminate transcription downstream of the T-DNA insertion [5]. Here, we performed transcript analysis by RT-PCR for the crwn3-1 and crwn4-1 alleles used in this study (Additional file 3). For crwn3-1, some transcript was detected downstream of the insertion; however, no transcript could be detected using primers that flanked the insertion. The crwn4-1 insertion blocked transcription downstream of the T-DNA. The lack of fulllength $C R W N$ transcripts from homozygous mutant lines indicates that all four mutations used in this study are likely to be loss-of-function alleles. We note that the $C R W N$ genes have similar developmental gene expression patterns: the steady-state abundance of transcripts for all four paralogs peak in proliferating tissues (http://bar.utoronto.ca/efp/cgi-bin/efpWeb.cgi) [18].

Mutant plants carrying single insertions were intercrossed and progeny carrying homozygous insertions in different combinations were recovered. Figure 2 shows the whole-plant phenotype of the viable mutants at the rosette stage just after the transition to flowering. Plants carrying a mutation in any single $C R W N$ gene had phenotypes similar to wild-type Columbia plants, as did the double crwn 2 crwn 3 and crwn3 crwn 4 mutants. The crwn 2 crwn 4 and crwn 1 crwn 4 double mutants exhibited slightly smaller rosettes, while the remaining double mutants, crwn 1 crwn 2 and crwn 1 crwn3, displayed markedly smaller rosette sizes. We were able to recover only two of the four triple mutants - crwn 1 crwn 2 crwn 4 and crwn 1 crwn 3 crwn4, both of which were extremely stunted and set few seed.

Our inability to isolate a mutant combining alleles in all four CRWN genes indicates that at least one functional CRWN protein is required for viability. Triple mutant plants carrying only CRWN2 or CRWN3 were extremely stunted, but still viable. This result suggests that CRWN2 or CRWN3 alone can cover the minimum requirements for the entire CRWN protein family. However, plants carrying only CRWN1 or only CRWN4 were not recovered, suggesting that CRWN1 and CRWN4 are specialized and that neither protein alone can express the full range of functions of the CRWN protein family. 


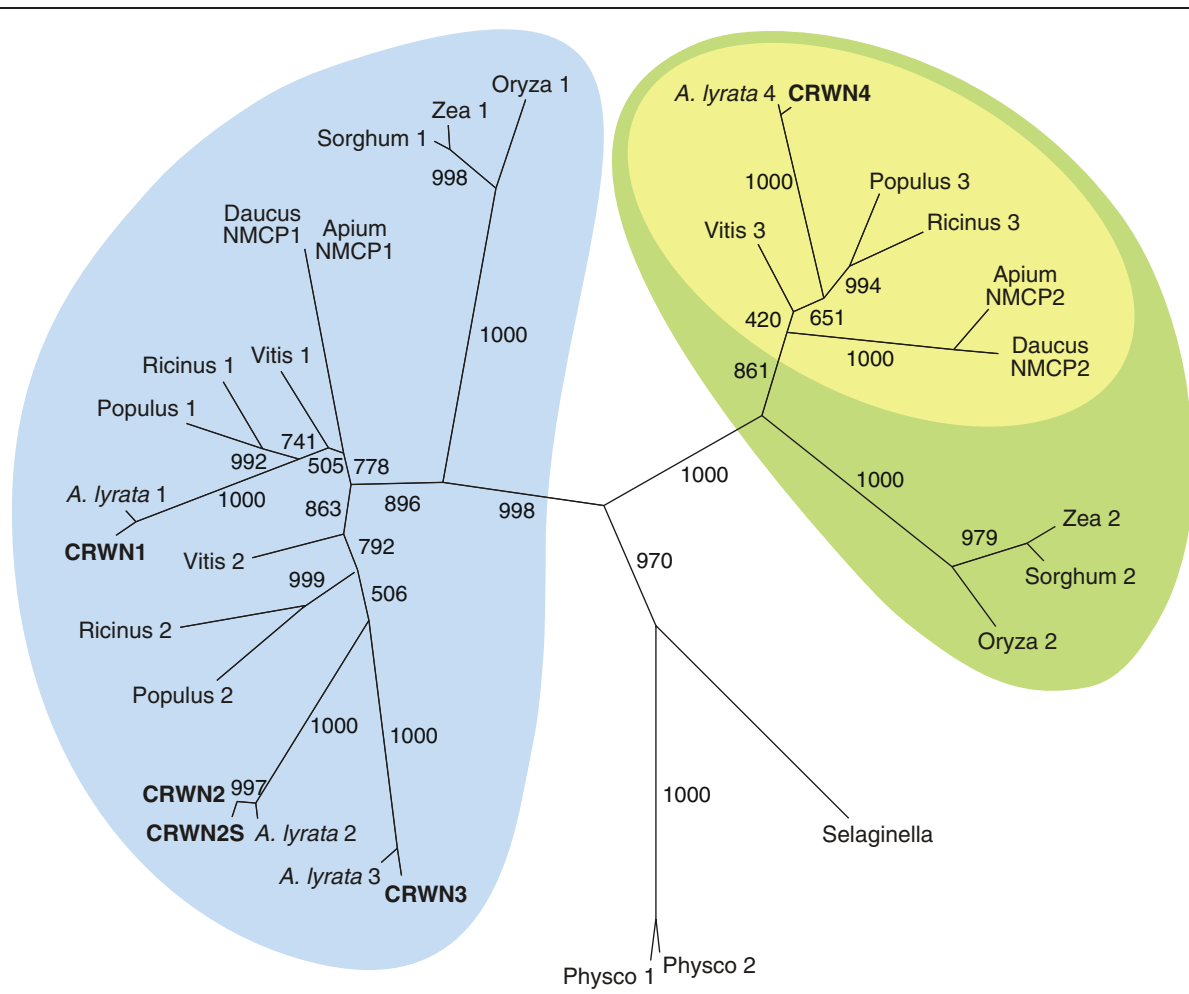

Figure 1 Phylogenetic relationships among CRWN proteins. A maximum likelihood tree of CRWN homologs constructed from an alignment of amino acid sequences that correspond to the coiled-coil domains. Bootstrap values (of 1000 replicates) are indicated on each branch. The A. thaliana CRWN proteins are indicated in bold, and homologs are labeled with the genus name and an assigned number (Additional file 1: Table S1). Two major clades are marked by blue and green; the yellow inset oval indicates a subgroup of CRWN4-like proteins from dicots that lack the conserved C-terminal domain (Additional file 2).

\section{CRWN proteins are required to maintain proper nuclear size and shape}

We next observed crwn mutant nuclei from adult leaf tissue to determine the role of different CRWN proteins in specifying nuclear size and shape. Among the single mutants, a deficiency of CRWN1 or CRWN4 reduced nuclear size (Figures 3 and 4A; Additional file 4), while loss of CRWN2 or CRWN3 had no effect. Combining a crwn1 mutation with a crwn 2 or crwn 3 mutation had a synergistic effect on nuclear size, suggesting that CRWN1 function overlaps, at

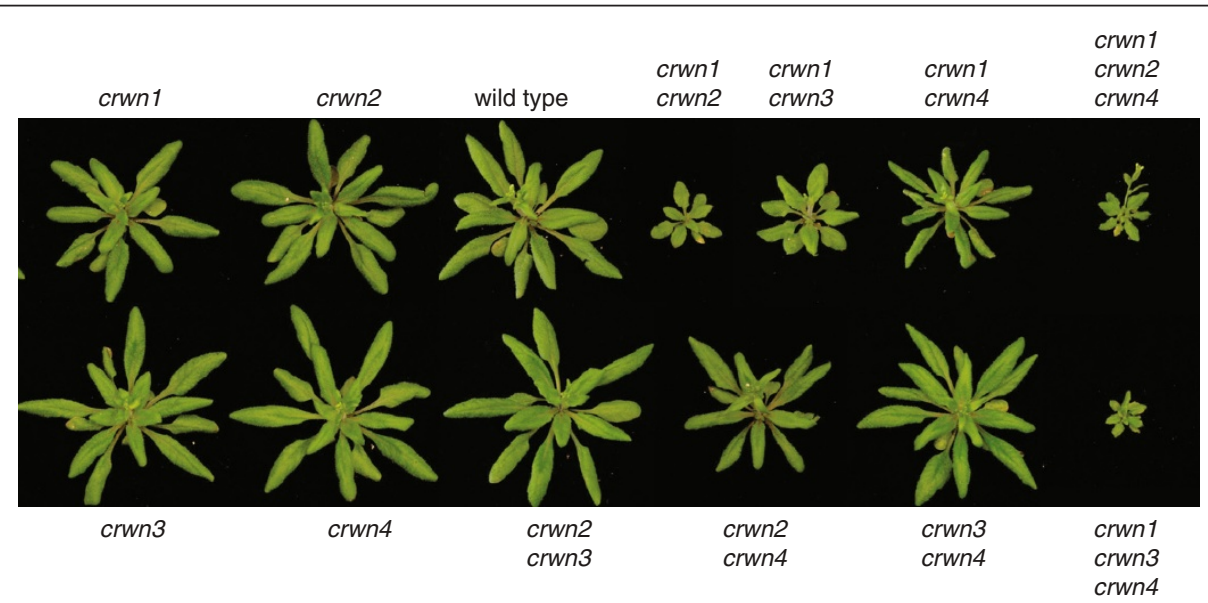

Figure 2 Whole plant phenotypes of crwn mutants. Leaf rosette structure of one month-old plants imaged just after initiation of flowering. Representative plants for the various genotypes are compared to a wild type (WT) Columbia plant. All plants were grown in parallel and photographed at the same magnification; the diameter of a WT rosette at flowering is ca. $8 \mathrm{~cm}$. 

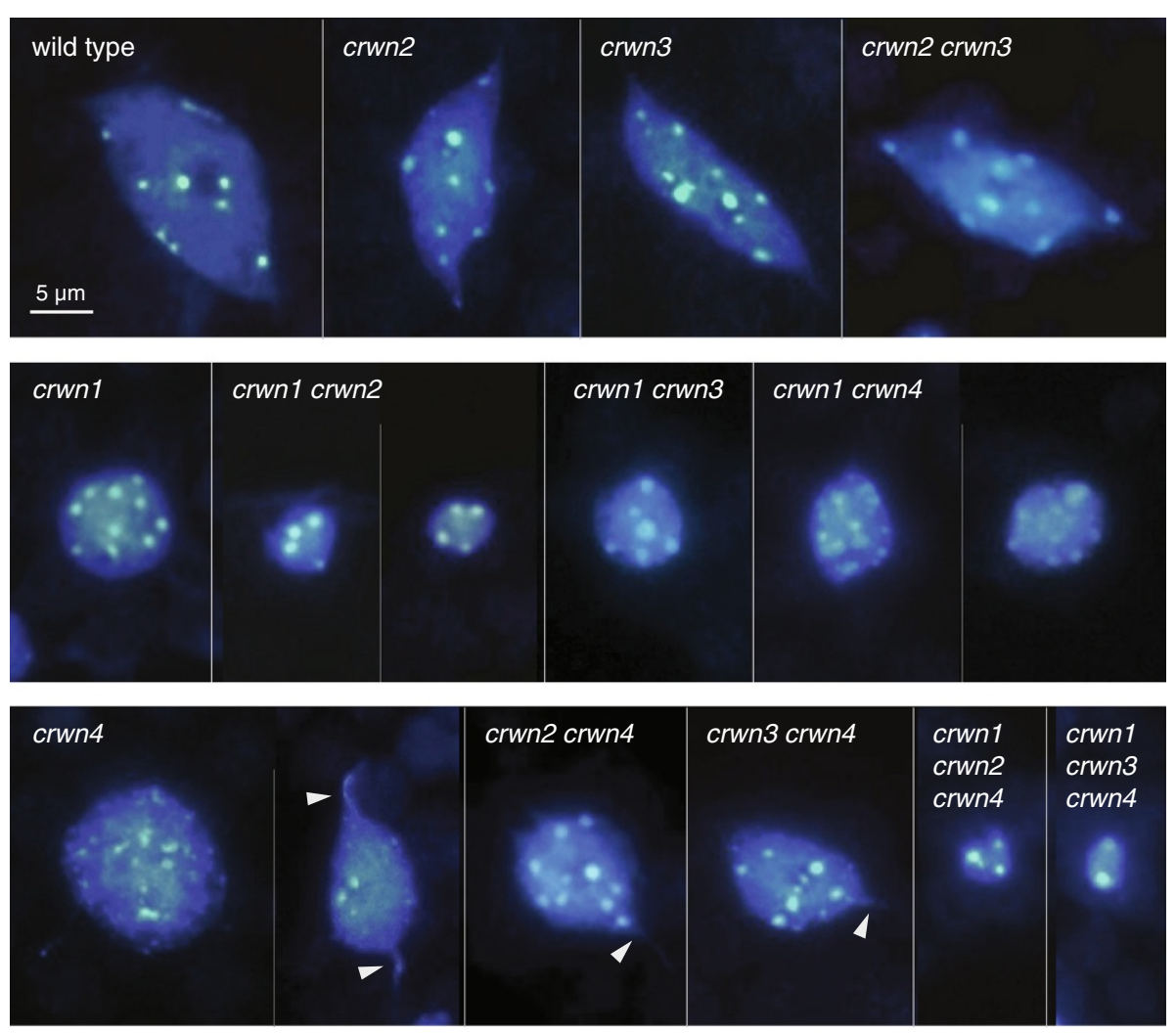

Figure 3 Nuclear phenotypes of crwn mutants. Images of representative DAPI-stained adult leaf cell nuclei from wild type and the twelve viable crwn mutants. The top row contains the wild type control and crwn2, crwn3 and crwn2 crwn3 mutants with normal nuclear morphology phenotypes. The middle row shows nuclear phenotypes of crwn 1 and double mutants containing a crwn 1 mutation. The bottom row displays nuclear phenotypes from crwn4 mutants, as well as higher-order mutants containing a crwn4 mutation. The arrowheads highlight thin projections from crwn 4 nuclei. A $5 \mu \mathrm{m}$ size bar is shown in the upper left inset; all images are shown at the same magnification.
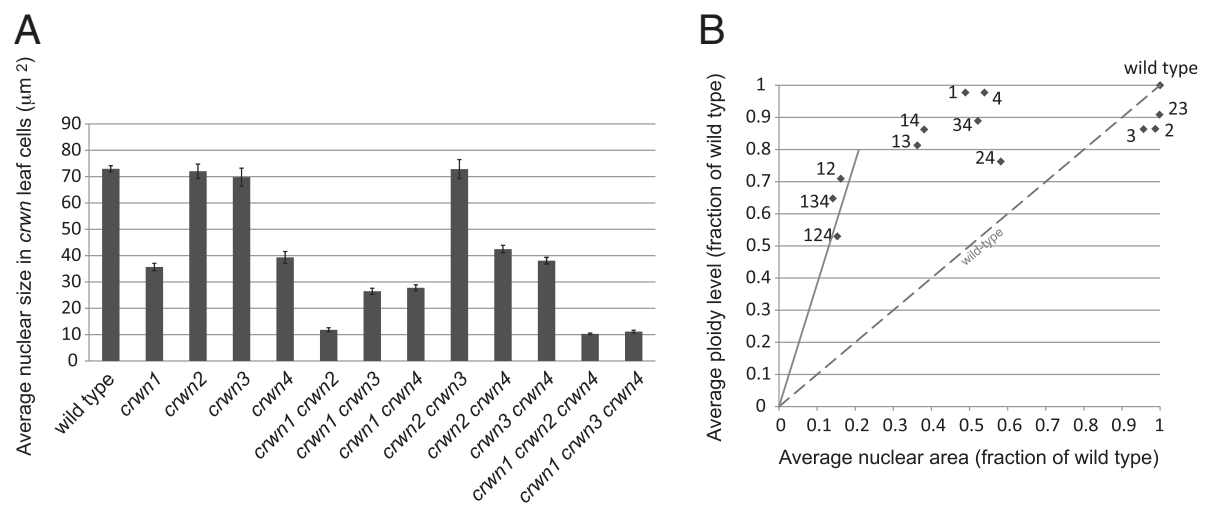

Figure 4 The effects of crwn mutations on nuclear size and nuclear DNA density in leaf cells. (A) Developmentally matched rosette leaves from approximately one month-old plants were fixed, cells isolated and stained with DAPI, and nuclei imaged using epifluorescence microscopy. The average areas of randomly-selected individual nuclei $(n=32-108)$ were determined for each genotype. Error bars indicated standard error of the mean. (B) Nuclear area measurements from panel A (see also, Additional file 4) were converted to relative values and were plotted against average endopolyploidy level for each genotype expressed as a fraction of the wild-type value. The average endopolyploidy levels (Additional file 4) were measured by flow cytometry using corresponding leaf samples. The diagonal dashed line indicates the expected linear relationship between nuclear area and endopolyploid level observed in wild-type plants. Note that panel A measures nuclear area but the isolated nuclei are flattened under a coverslip to a uniform thickness (see Additional file 6) and therefore nuclear area is proportional to and therefore a proxy for nuclear volume. The numbers next to the symbols indicate the corresponding crwn genotype. Data points above the dashed line indicate an elevated nuclear DNA density relative to wild type. The solid line corresponds to a nuclear DNA density four-times that of wild type. 
least partially, with those of CRWN2 and CRWN3. Double mutant combinations containing crwn 4 and either crwn 2 or crwn3 did not show additive phenotypes but rather resembled crwn4. In contrast, combination of a crwn 1 with a crwn 4 mutation had an additive effect on nuclear size. These findings indicate that CRWN1 and CRWN4 are the major determinants of nuclear size among the CRWN paralogs. Further, the additive effects of crwn1 and crwn 4 mutations suggest CRWN4 acts independently from CRWN1, consistent with their distinct phylogenetic grouping (Figure 1) and the genetic analysis shown in Figure 2.

CRWN proteins are required for development or maintenance of the elongated spindle shapes which characterize larger nuclei in differentiated wild-type cells [19]. We previously reported that a deficiency of CRWN1 causes nuclei in all cells to adopt the spherical shape characteristic of proliferating tissue at root and shoot apices [5]. The present study confirmed the importance of CRWN1 for nuclear shape differentiation and also uncovered a similar role for CRWN4 (Figure 3), a conclusion also reached recently by Sakamoto and Takagi [7]. Nuclei from crwn4 leaf tissue often have irregular margins and are more spherically shaped, compared to wild-type nuclei (Additional file 5). However, crwn4 nuclei are less uniformly round in comparison to $c r w n 1$ nuclei, particularly larger crwn 4 nuclei. Further, crwn4 nuclei occasionally contain thin projections that appear to be drawn from the nuclear surface (arrowheads in Figure 3).

\section{Loss of CRWN proteins affects nuclear DNA packing density}

The direct correlation between endopolyploidy and nuclear size in wild-type Arabidopsis cells [20] prompted us to examine this relationship within the crwn mutants. We measured the average endopolyploidy level of nuclei from the same adult leaves harvested for the nuclear size analysis shown in Figure 4A (see also Additional file 4). Some crwn mutants showed a decrease in endopolyploid levels, particularly the crwn triple mutants and the crwn 1 crwn 2 double mutant, but the remaining crwn genotypes had average endopolyploidy levels that approached wildtype levels (Figure 4B). The dashed line in Figure 4B depicts the expected nuclear size change in response to a reduction in endopolyploidy based on the established one-to-one relationship between nuclear volume (approximated by nuclear area in our measurements of isolated and flattened leaf cell nuclei, Additional file 6) and DNA content in wild type plants. With the exception of crwn 2 and crwn 3 , the crwn mutations caused a more pronounced reduction in nuclear size than predicted from the observed endopolyploidy level. As a consequence, crwn mutants display a spectrum of nuclear DNA densities, ranging from wild-type values in crwn2 and crwn 3 mutants to four-fold higher densities in crwn 1 crwn 2 double mutants and the two viable crwn triple mutants.

We then investigated the relationship between nuclear size and DNA content by examining the effects of different crwn genotypes on nuclear size in leaf guard cells, a diploid cell type where endopolyploidy is not a factor [21]. crwn1 mutant guard cell nuclei were smaller than nuclei in wild type cells with an area approximately onehalf of the wild type value, corresponding to a volume difference of approximately threefold assuming a roughly spherical shape to nuclei in the cell (Figure 5). Double and triple mutants lacking CRWN1 displayed nuclear sizes similar to the crwn 1 single mutant. Consistent with their effects on nuclear size shown in Figure 4A, neither the crwn 2 nor crwn 3 mutation affected nuclear size in guard cells. Interestingly, the size of nuclei in crwn4 guard cells was also unaffected, in contrast to the effect seen in a population of adult leaf cells (Figure 4A). However, crwn 2 crwn 3 , crwn 2 crwn4, and crwn 3 crwn 4 double mutants had nuclei approximately $20 \%$ smaller than those seen in wild-type guard cells, suggesting some functional redundancy among CRWN2, CRWN3 and CRWN4 proteins. Overall, our results indicate that CRWN1 plays the major role in affecting nuclear size in the absence of changes in endopolyploidy.

\section{CRWN4 maintains interphase chromocenter integrity and organization}

Considering the dramatic effects of crwn mutations on nuclear size and morphology, we turned our attention to the role of CRWN proteins on the internal organization of the nucleus. A conspicuous feature of Arabidopsis interphase nuclei are discrete foci of heterochromatin, or chromocenters, visualized as bright spots after staining with fluorescent DNA-intercalating dyes [22]. A typical interphase nucleus contains approximately ten chromocenters corresponding to the number of diploid chromosomes $(2 \mathrm{n}=10)$ [23]. Chromocenter number remains fairly constant over a wide range of nuclear sizes and endopolyploid levels ( $2 \mathrm{n}$ to $16 \mathrm{n}$ ), most likely as a result of lateral association of sister chromatids after endoreduplication [24,25]. We found that the average chromocenter number in crwn1, crwn2 and crwn3 leaf cell nuclei was similar to that seen in wild-type leaf cell nuclei (Figure 6A) and did not change dramatically as a function of nuclear size. In crwn4 nuclei, however, chromocenter number was strongly correlated with nuclear size (Figure 6A): smaller nuclei contained fewer chromocenters than the wild-type value of $\sim 9$, while larger, presumably endopolyploid, $c r w n 4$ nuclei exhibited a wide range of chromocenter numbers (2-27). A similar pattern was observed in double mutants containing the crwn 4 mutation (Additional file 7). In contrast, double mutants containing the crwn1 allele paired with another crwn mutation displayed a reduced average 


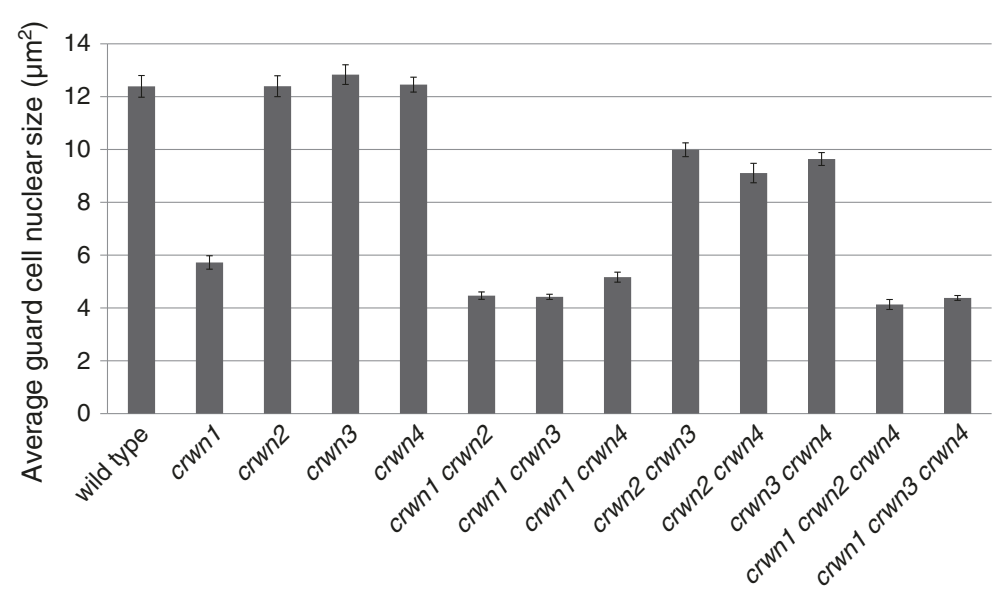

Figure 5 Average leaf guard cell nuclear sizes in crwn mutants. Two week-old plants were harvested, fixed, stained with DAPI, and leaf guard cell nuclei were imaged using confocal laser scanning microscopy. The area of randomly-selected individual nuclei $(n=19-29)$ were determined for each genotype. Error bars indicated standard error of the mean.

chromocenter number with a weaker association with nuclear size (Figure 6A and Additional file 7).

To explore the chromocenter phenotype in more detail, we developed a statistic, referred to as an aggregation index (AI) (see Methods), to characterize the distribution of visible DAPI-bright spots within interphase nuclei. The value of this index ranges from 0 to 1 , reflecting both the number of distinct chromocenter spots and the uniformity of their size distribution. The expected AI for wild-type nuclei containing 10 equally sized chromocenters is 0.1 , while clustering of chromocenters into fewer but larger aggregates will lead to a higher AI value. A dispersal of chromocenters into smaller heterochromatic satellites will push the AI lower. For a given chromocenter number, a skewed CC size distribution is associated with a larger AI compared to when each $\mathrm{CC}$ is equally sized. As shown in Figure 6B, the $\mathrm{AI}$ index of wild-type nuclei averaged close to 0.1 and was not affected significantly by nuclear size. The absence of a significant correlation between AI and nuclear size indicates that chromocenter organization remains constant across different endopolyploidy levels in wild-type nuclei. A similar pattern was observed for the crwn1, crwn2, and crwn 3 mutant samples. In contrast, combining crwn 1 and crwn 2 mutations led to an approximately two-fold higher AI over a range of nuclear sizes, consistent with the two-fold reduction in chromocenter number via aggregation in crwn1 crwn 2 mutants. A different pattern was displayed in the crwn 4 sample, which displayed a negative correlation between AI and nuclear size. This result suggests a tendency for chromocenters to aggregate in smaller crwn4 nuclei and to become dispersed in larger crwn 4 nuclei. The reduction in chromocenter number in crwn 1 crwn 2 and crwn 4 mutants with smaller nuclei could reflect the aggregation of individual chromocenters in the limited confines of these nuclei, but a similar clustering does not occur in small wild-type nuclei, arguing that small nuclear dimensions alone are insufficient to cause clustering. The variability in chromocenter size and number in crwn mutant nuclei suggests that CRWN proteins are required for proper organization of heterochromatin in interphase nuclei.

We tested this hypothesis by visualizing the spatial arrangement of chromocenter-associated genomic regions in crwn 1 crwn 2 and crwn 4 mutants. Arabidopsis chromocenters are comprised of large segments of repetitive DNA such as the tandemly-arrayed centromeric and 5S RNA repeats located within pericentromeric regions [23]. Using fluorescent in situ hybridization (FISH), we examined the spatial organization of the major 180-bp centromeric tandem repeat and the 5S RNA gene arrays in both large and small nuclei from wild-type, crwn1 crwn 2 and crwn4 plants (Figure 7A, B). The centromeric and 5S RNA repeats were co-localized with the DAPIbright spots in both small and large wild-type nuclei, confirming previous reports that these sequences are normally compartmentalized within chromocenters at the nuclear periphery [26] (see Additional file 8: Movie S1). It was common to find a decondensed centromere signal at several chromocenters in wild-type nuclei; however, decondensed centromeric repeat clusters were infrequently observed in crwn 1 crwn 2 nuclei and the total number of clusters was reduced (Figure 7C) (also see Additional file 8: Movie S2). These findings indicate that there is a compaction of the centromere repeat arrays within coalesced chromocenters in crwn 1 crwn 2 nuclei. In contrast, the number of discrete centromere repeat clusters visible in crwn4 nuclei was more variable, and decondensed signals were often seen in nuclei with numerous clusters. This pattern is consistent with the 

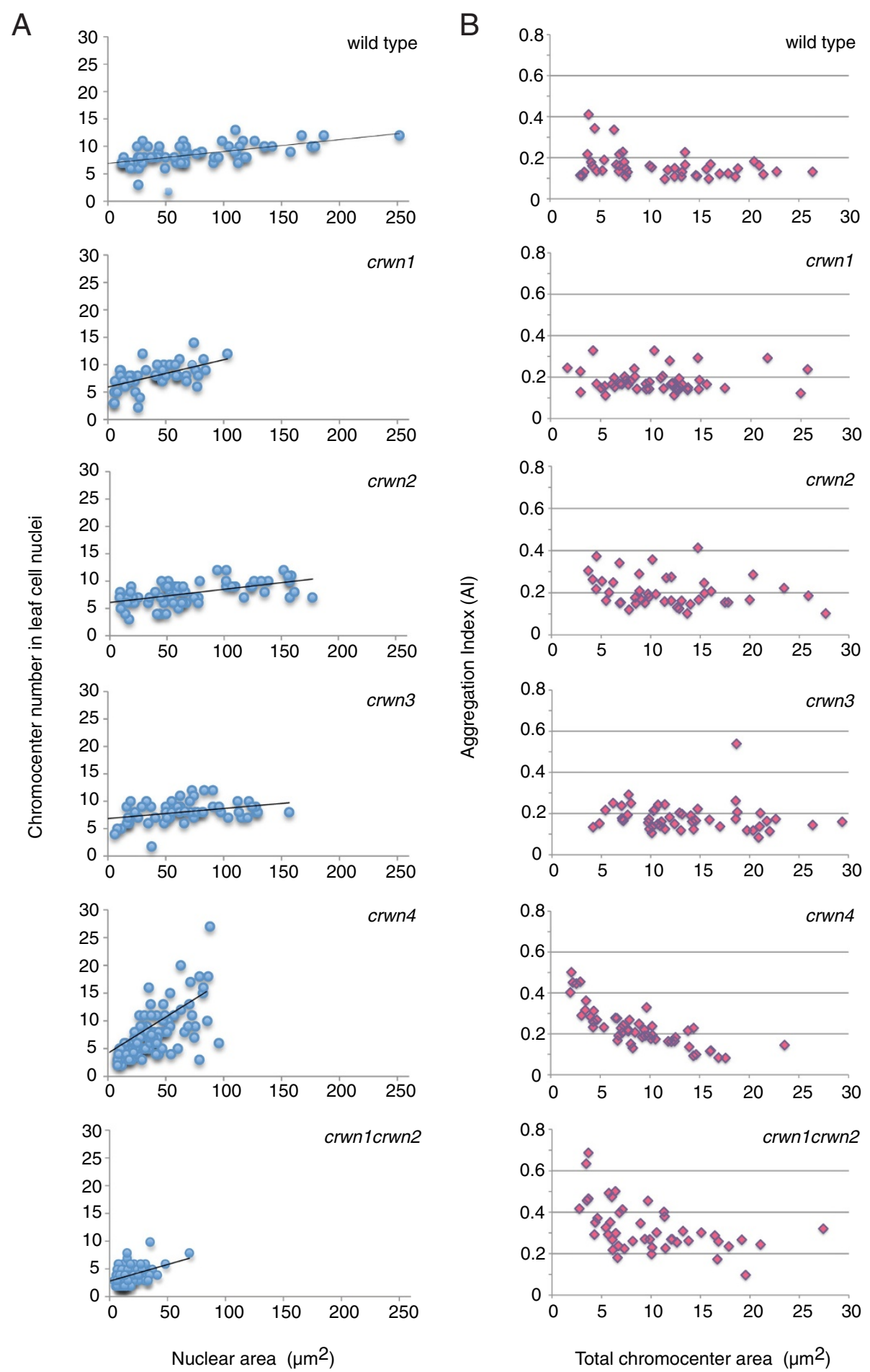

Figure 6 Chromocenter morphology changes in crwn mutants. (A) Nuclei were imaged from developmentally matched rosette leaves from approximately one month-old plants, cells isolated and stained with DAPI, and nuclei imaged using epifluorescence microscopy. The area and chromocenter number of randomly-selected individual nuclei $(n=47-132)$ were determined for each genotype, and chromocenter number was plotted against nuclear area. A linear regression line showing the relationship between chromocenter number and nuclear size (as a proxy for endopolyploidy level) was plotted for each genotype. (B) Nuclei were imaged fully expanded rosette leaves from approximately one month-old plants and the chromocenter Aggregation Index (Al) was plotted against the total chromocenter area $\left(\mu \mathrm{m}^{2}\right)$. 
A

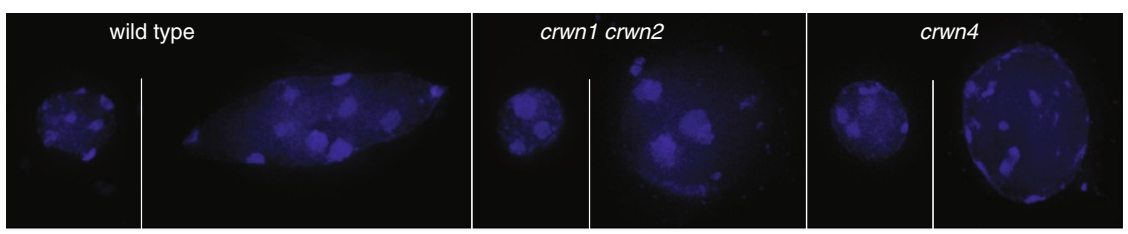

B

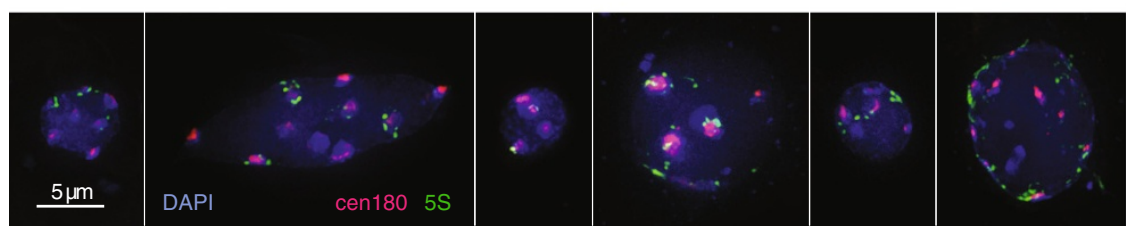

C

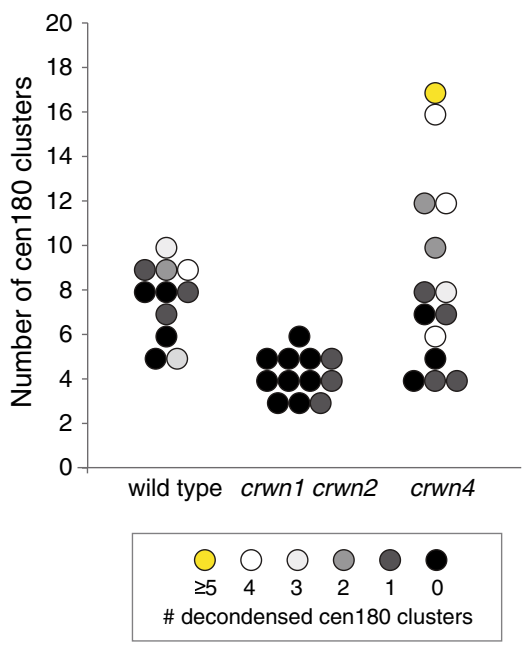

D

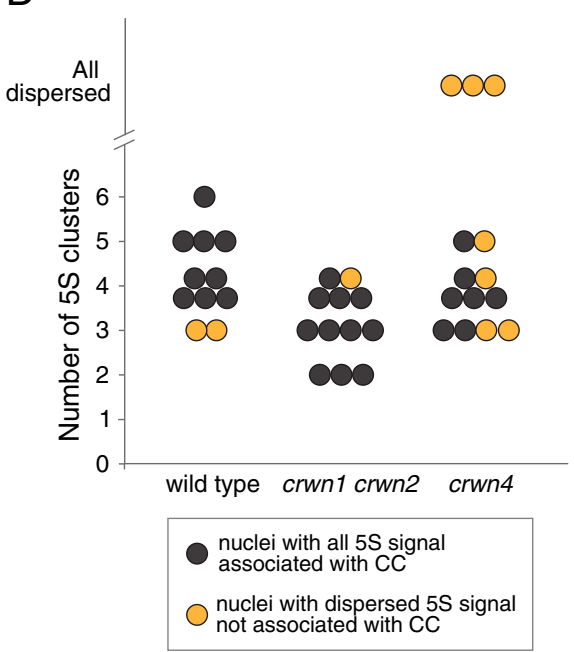

Figure 7 Chromocenter organization is altered in crwn1 crwn2 and crwn4 mutants. (A and B) Fluorescence in situ hybridization of representative small and large nuclei from wild type, crwn1 crwn2 and crwn4 cells prepared from leaves of adult plants after bolting. Blue: DAPI, pink: 180-bp centromere repeats, green: $5 S$ RNA genes, bar, 5 Mm. (C) The distribution of nuclei based on the number of centromere repeat clusters in different genotypes. Each circle represents an individual nucleus in the FISH experiment. The color indicates the level of decondensation of the centromere signal. (D) The distribution of nuclei based on the number of 55 RNA repeat clusters in different genotypes. Each circle represents an individual nucleus. The color indicates the level of dispersion of the $5 \mathrm{~S}$ repeat signal.

hypothesis that chromocenters become dispersed in larger crwn4 nuclei. A similar but more pronounced trend was seen for the 5S RNA gene arrays (Figure 7B, D), which were dispersed outside chromocenter aggregates in roughly one-half of the crwn4 nuclei. We note that the dispersed 5S RNA gene signal remained localized to the nuclear periphery (see Additional file 8: Movie S3). The apparent dispersal of chromocenters in larger crwn 4 nuclei and the mis-positioning of centromeric and $5 \mathrm{~S}$ RNA repeats outside of the chromocenter indicates that higher-order organization of heterochromatin breaks down in interphase in the absence of CRWN4.

\section{Discussion}

Our results demonstrate that the CRWN family is essential for viability in Arabidopsis and required for proper nuclear organization. Redundancy and diversification exists among the four CRWN paralogs, which belong to a plant- specific family of nuclear coiled-coil proteins that forms two clades: one including CRWN1, CRWN2 and CRWN3 and the other containing CRWN4 (Figure 1). The divergence of CRWN4 relative to the other CRWN paralogs, also reported by Kimura et al. (2010) [10] and Ciska et al. (2013) [9], is supported by our genetic analysis. First, the inviability of triple mutants containing either CRWN1 or CRWN4 alone indicates that CRWN1 and CRWN4 possess non-overlapping functions (Figure 2). Second, loss-of-function mutations in CRWN1 and CRWN4 have distinct phenotypes; for example, crwn 1 is the only crwn mutation that affects nuclear size in diploid guard cells (Figure 5), while the dispersed chromocenter phenotype is unique to the crwn4 mutant (Figures 3 and 7). Finally, the functional distinction between the two CRWN clades is supported by taxa, such as rice and maize, which contain only two CRWN-like proteins: one CRWN1-like and one CRWN4-like. 
The phenotypic effects of combining mutations in different $C R W N$ genes demonstrates that all four paralogs are involved in specifying nuclear size in adult cells. A deficiency in CRWN1 led to a dramatic reduction in nuclear size independent of an effect on endopolyploidy (Figure 4). Loss of CRWN4 also reduced nuclear size in leaf cells (Figure 4) as was recently shown by Sakamoto and Takagi (2013) [7]. Interestingly, crwn4 nuclei in leaf guard cells were not reduced in size (Figure 5), despite the fact that CRWN4 is expressed in this cell type (http://bar.utoronto. ca/efp/cgi-bin/efpWeb.cgi) [18], suggesting cell-type specific requirements exist for different CRWN proteins. Combining a crwn1 loss-of-function mutation with a deficiency in any of the remaining three paralogs causes a further reduction in nuclear size in leaf cells. The additive effect on nuclear size indicates that CRWN2 and CRWN3 share overlapping functions with CRWN1, although loss of CRWN2 and/or CRWN3 has no effect on nuclear size or endopolyploidy in leaf cells when a functional $C R W N 1$ allele is present. We previously reported that the crwn2-1 allele caused a reduction in nuclear size in leaf cells [5]; the reason for the different behavior displayed in this study is unknown, but we have noted variability in nuclear phenotypes among different crwn 2 mutant lines. Regardless, it is clear that CRWN1 plays the major role in adult leaf tissue among the three paralogs in the CRWN1-like clade. This situation might reflect the higher level of expression of CRWN1 in leaf tissue compared to CRWN2 and CRWN3 - ca. 30 FPKM (fragment per kilobase/million mapped RNA-seq reads) for CRWN1, with $4 \times$ and $2 \times$ less expression from CRWN2 and $C R W N 3$, respectively (data not shown). Alternatively, the different contributions of CRWN1-like genes could also result from structural differences among the encoded proteins.

Our data indicate that the primary phenotype of crwn mutants is a reduction in nuclear size and that the reduction in endopolyploidy observed in double and triple crwn mutants is a secondary effect in response to reduced nuclear size. First, mutation of either CRWN1 or CRWN4 had an effect on nuclear size in leaf cells without affecting endopolyploidy (Figure 4) (see also [7]). Second, effects on endopolyploid levels were only seen in mutants that contained two or more crwn mutations and exhibited severely reduced nuclear size. These considerations indicate that loss of CRWN activity alters the relationship between DNA content and nuclear volume, leading to a higher than normal nuclear DNA density. In the most severely affected mutants, the nuclear DNA density reaches four times the level seen in wild-type cells. It is intriguing that the crwn mutants with the most abnormal whole-plant dwarfing phenotypes, including crwn 1 crwn 2 and the viable triple crwn mutants, are the ones with the highest average nuclear DNA density.
We hypothesize that the CRWN proteins are required for nuclear expansion after nuclear reformation in telophase [27-29], and that the loss of these proteins, especially in combination, results in an elevated nuclear DNA density. Evidence supporting this mechanism comes from a recent report demonstrating that crwn 1 crwn 2 cotyledon nuclei expand more slowly than their wild type counterparts during the first 72 hours of seed germination, a period normally characterized by a ca. 10-fold expansion of nuclear size in the absence of endoreduplication [30]. Interestingly, the rate of contraction of crwn 1 crwn 2 embryonic cotyledon nuclei during seed maturation is also reduced relative to wild type. These observations suggest that CRWN proteins are involved in remodeling nuclear structure during interphase in response to developmental and environmental cues. Similarly, a constraint in nuclear expansion associated with endoreduplication [20,31] could explain the limit on endopolyploid levels observed in high-order crwn mutants.

The large coiled-coil domains that comprise the central region of all four CRWN proteins point toward a structural role in the nucleus. Recent analysis based on secondary structure for analogues of Arabidopsis coiledcoil proteins indicates that CRWN proteins are candidates for paramyosin homologues [11]. Paramyosin is a structural protein found in invertebrate muscles, where it forms the core of thick filaments and bundles with myosin motors. Deficiency of paramyosin, encoded by the unc-15 gene in C. elegans, leads to shortened, misformed and apparently fragile thick filaments in the nematode's muscles [32]. The structural similarity to paramyosin suggests models for CRWN action as architectural components of the nucleoskeleton. Models of this type predict that crwn mutant nuclei would have a less sound structural foundation and be more prone to breakage or distortion by intracellular forces (e.g., exerted by the cytoskeleton or at programmed nuclear expansion/contraction transitions), as seen in animal cells when lamin is disrupted [33] or down-regulated [34]. The irregular margins and thin projections seen among crwn4 nuclei are consistent with this structural integrity model. These predictions, however, are not consistent with the smaller, round nuclei in crwn1 mutants that do not adopt the elongated spindle shapes typical of wild-type nuclei in many cell types. These considerations suggest alternative models wherein the CRWN1 protein might establish flexible hinge regions or expansion zones in the nucleoskeleton, facilitating nuclear size changes that accompany endopolyploidy and other developmental transitions. We note that both nucleoporin $136[35,36]$ and LINC complex mutants [15] in Arabidopsis also lead to more spherical nuclear shapes, suggesting that CRWN proteins might interact with these complexes at the nuclear periphery. 
Deficiency of CRWN proteins also affects chromocenter organization (Figures 6 and 7). We previously reported that chromocenter number decreases approximately twofold in crwn 1 crwn 2 nuclei [5], and we confirmed these results here while extending our analysis to the entire $C R W N$ gene family. One unexpected finding was the wide variation in chromocenter number in crwn 4 nuclei and the direct correlation between chromocenter number and nuclear size. The reduction in chromocenter number in crwn1 crwn 2 mutants, as well as crwn 4 mutants with smaller nuclei, is consistent with aggregation of individual chromocenters. The in situ hybridization data shown in Figure 7 further support this conclusion. Further, we demonstrated that chromocenter organization was disrupted in larger crwn 4 nuclei as evidenced by the dispersed signals seen for the 5S RNA genes, and to a less extent, the centromere repeat arrays. Considering that chromocenters are localized primarily to the nuclear periphery [23,26] (see Additional file 8: Movies S1-3) where CRWN proteins are also located [5-7], CRWN proteins might play a direct role in ensuring proper heterochromatin organization. In such models, CRWN1 and CRWN2 would act to prevent chromocenter aggregation and CRWN4 would exert a complementary effect to maintain chromocenter integrity.

The four distinct phenotypes displayed by crwn mutants - reduced nuclear size, altered nuclear shape, elevated nuclear DNA density and abnormal organization of constitutive heterochromatin - highlight a functional connection among these different aspects of nuclear architecture. The lack of whole-plant phenotypes of Arabidopsis crwn1 and crwn 4 mutants is remarkable in light of the dramatic nuclear changes occurring in these mutants and underscores plants' plasticity in their ability to execute an apparently normal developmental program in spite of these nuclear changes. The diversity of CRWN proteins and the ability to work with viable crwn mutants that exhibit dramatic nuclear phenotypes will facilitate the elucidation of the mechanisms through which these essential proteins exert their effects on nuclear organization.

\section{Conclusions}

This study addresses fundamental questions about how plant cells specify and control the morphology of their nuclei and its relationship with internal chromatin organization. We conducted a comprehensive reverse genetics study of the CRWN gene family in Arabidopsis, which encode NMCP-class proteins implicated in nuclear morphology and organization. We demonstrated that CRWN proteins are essential for viability, and in the process, uncovered a surprisingly high degree of functional diversity among the CRWN proteins. CRWN1 and CRWN4 are the major determinants of nuclear size and shape, and we hypothesize that deficiency in CRWN proteins leads to defects in nuclear expansion and remodeling. One consequence of this deficiency is an increase in nuclear DNA density as endoreduplication levels are not affected except in the most extreme cases (e.g., crwn 1 crwn 2 and the viable triple mutants). Our findings also demonstrated that CRWN4 plays a role in maintenance of heterochromatin organization in interphase nuclei. The specificity of the nuclear morphological and higher-order chromatin organization defects seen in crwn mutants reveals the interplay between nuclear morphology and the three-dimensional packaging of the genome.

\section{Methods}

\section{Plant materials and growth conditions}

All T-DNA insertion alleles used in this study are from the SALK collection [17] in strain Columbia, and single mutant lines were originally obtained from the Arabidopsis Biological Resource Center (ABRC) at The Ohio State University. Plants were grown in long-day lighting conditions ( $16 \mathrm{~h}$ of light $/ 8 \mathrm{~h}$ of dark) at $23^{\circ} \mathrm{C}$ on soil (Metro-Mix 360, SunGro, Vancouver) in environmental growth chambers. Genotyping of individual T-DNA alleles was performed by standard PCR using the following pairs of allele-specific primers: SALK_025347 (crwn1-1), 5'-TGC CTT CTC CTC GCT TTT CAA-3' and 5' -TGC GTG AAT GGG AAA GAA AGT TG-3'; SALK_076653 (crwn2-1), 5'-GAA GCT CAT TGC TAG AGA AGG GG-3' and 5' -AAC GCT GAT CGT TCA TGT TCC A3'; SALK_099283 (crwn3-1), 5'-TTC TGC ATC TTG ACA CCA TCC AA-3' and 5'-TCG TCG ACT AGT TAA CAA AAT CA-3'; SALK_079296 (crwn4-1), 5' -CGC AAA GCC TTC GAA GAC AAA-3' and 5'-GCT TCA GCC AGC ATT TCA AGC-3'.

\section{Phylogenetic tree construction}

Amino acids similar to CRWN1 were downloaded from public databases (see Additional file 1: Table S1). The program ClustalX was used to align the amino acid sequences. The tree in Figure 1 is based on an alignment of the region of highest conservation across all amino acid sequences, corresponding to the coiled-coil domains (amino acids 64 to 651 in CRWN1). A maximum likelihood tree was constructed using Phylip 3.69 with 1000 bootstrap replicates.

\section{Diploid guard cell nuclear area measurement}

Two-week old seedlings were harvested and fixed in 3:1 acetic acid:ethanol. Nuclei in the fixed tissue were stained using DAPI (10 $\mu \mathrm{g} / \mathrm{ml}, 2$ minutes), and guard cell nuclei were imaged using laser scanning confocal microscopy (Leica SP5). Images were taken at the focal plane with the maximum nuclear area, and the resulting images were processed using ImageJ software. 


\section{Leaf nuclei isolation and imaging}

Nuclei were isolated from the fifth true leaf of adult plants harvested after initiation of flowering stem elongation (stem height $\geq$ vegetative rosette diameter). Therefore, the tissues were developmentally matched across the different genotypes. Mesophyll cells predominated but other cell types were present. Each harvested leaf was bisected and one half used for the nuclear area measurement, while the remaining half leaf was processed for flow cytometry measurements (see below). Leaf tissue was fixed using a 3:1 acetic acid:ethanol solution and tissues were rehydrated in $100 \mathrm{mM}$ sodium citrate buffer $\mathrm{pH} 4.8$ for 15 min followed by incubation in digestion buffer $(0.03 \%$ cytohelicase, $0.03 \%$ pectolyase, and $0.03 \%$ cellulase Onozuka RS in $100 \mathrm{mM}$ sodium citrate buffer pH 4.8) for 2 hours at $37^{\circ} \mathrm{C}$. Digested tissue was carefully homogenized by pipetting, centrifuged briefly at low speed, and resuspended with $100 \mathrm{mM}$ sodium citrate buffer $\mathrm{pH} 4.8$; this cycle was repeated three times and the final pellet was resuspended in 3:1 acetic acid: ethanol. The resulting suspension of nuclei were pipetted onto microscope slides, dried for ca. $1 \mathrm{~min}$, and stained with $10 \mu \mathrm{g} / \mathrm{ml}$ 4',6-diamidino-2-phenylindole (DAPI). A Leica DM 5500 epifluorescence microscope was used to image the nuclei, and the nuclear area was measured from digital images using ImageJ software after manual tracing of nuclear boundaries. Note that the chromocenter number versus leaf cell nuclear area scatter plots shown in Figure 6A were generated in a separate experiment from the one shown in Figure 4, but developmentally matched leaf tissues were harvested as described above.

\section{Aggregation index measurement}

Tissue from adult leaves was harvested and prepared as described for FISH (see below). Rather than performing the in situ hybridization step, nuclei were stained with DAPI and imaged using optical sectioning microcopy. Projections of the processed images were analyzed using ImageJ software to identify chromocenters. Briefly, the images were manually manipulated when necessary to adjust the local threshold and chromocenter area and number were assigned using the Analyze Particle function of the software. The aggregation index (AI) was calculated using the following equation: $A I=\Sigma\left(S_{i} / S_{\text {total }}\right)^{2}$; where $i=1, \ldots, n$; $\mathrm{S}_{\mathrm{i}}=$ the area of chromocenter $\mathrm{i} ; \mathrm{S}_{\text {total }}=$ the total area of all chromocenters in the nucleus.

\section{Flow cytometry}

Bisected tissue from the fifth true leaf of adult plants were harvested (see above) and immersed in magnesium sulfate buffer [37] and chopped with razor blades in a petri dish. The resulting suspension was filtered through a nylon mesh (diameter $=30 \mu \mathrm{m}$; Partec Cell Trics ${ }^{\oplus}$,
Münster, Germany). The nuclear suspension was incubated with RNAse A (Ribonuclease A, from bovine pancreas, Sigma, St. Louis, MO, USA) $(50 \mu \mathrm{g} / \mathrm{ml})$ on ice for $15 \mathrm{~min}$ and stained with propidium iodide $(50 \mu \mathrm{l} / \mathrm{ml})$ in the dark for 6 hours. Average ploidy level for each genotype was calculated based on the peaks generated from an analytical flow cytometer (Accuri 6 model, Accuri cytometers, Ann Arbor, MI, USA).

\section{Fluorescent in situ hybridization (FISH)}

Fully expanded adult leaf tissue was harvested less than a week after flowering stem elongation, and fixed in Buffer A [38] with 4\% formaldehyde at room temperature with agitation for $>1$ hour. After rinsing with Buffer A, the tissue was chopped repeatedly with razor blades until a homogenous texture was achieved. A clear nuclear suspension was pipetted from the leaf debris and used for fluorescence in situ hybridization as described by Golubovskaya et al. (2002) [38]. The centromere probe 5' - Cy5GGTTGCGGTTTAAGTTCTTATACTCAATC -3' was synthesized by Integrated DNA Technologies (Coralsville, IA, USA), and the $5 \mathrm{~S}$ probe was amplified from genomic DNA using primers $5^{\prime}$-CTNCCNGGNAGNTCACCC-3' and 5'-CCTNGTGNTGNANCCCTC-3', followed by labeling using a nick translation protocol and Rhodamine labeled dCTP (Roche; Indianapolis, IN, USA).

\section{Additional files}

Additional file 1: Table S1. CRWN-like proteins used in this study. The
first column shows the abbreviated name of the protein used in
alignment to construct the tree shown in Figure 1. The remaining
columns indicate the identity and the source of each protein sequence.
Additional file 2: Amino acid sequences comprising the extreme
C-termini of 28 CRWN-like proteins, including ten CRWN4-like
proteins. The similarity in this region, which falls outside of the coiled-
coil domains, reinforces the topology of the tree shown in Figure 1. All of
the proteins within the CRWN1-like clade, as well as the Physcomitrella
homologs, contain a conserved C-terminal motif and a group of acidic
residues approximately 25 amino acids from the end of the protein.
Monocot CRWN4-like proteins contain a region with similar features but
these conserved motifs are absent in CRWN4-like proteins from dicots
(denoted by the yellow oval in Figure 1).
Additional file 3: Transcript analysis of the crwn3-1 and crwn4-1
alleles used in this study. Reverse transcription-PCR results investigating
the effect of T-DNA insertions on the transcription of CRWN3 and CRWN4.
Panel A shows that a CRWN3 transcript is produced from the wild-type
allele but not from the crwn3-1 allele using primers spanning the T-DNA
insertion site. Panel B demonstrates that some transcription can be
detected downstream of the insertion site from the crwn3-1 allele using
RT-PCR and a primer set recognizing sequences 3 ' of the insertion site.
Panel C indicates that the T-DNA insertion in the crwn4-1 allele blocks
transcription. Amplification of CDNA from cyclophilin and Actin2 were
used as positive controls. M, marker lanes; + RT (plus reverse transcriptase);
- RT (no reverse transcriptase). Information on the oligonucleotide primers
used in these experiments is shown at the bottom of the figure. Our
previous data [5] indicated that the crwn 1-1 and crwn2-1 alleles block
transcription downstream of the T-DNA insertion site in the sixth exon of
both genes.


Additional file 4: Table S2. The nuclear phenotype data for crwn mutants used to construct Figure 4 is displayed in tabular form. The average endopolyploid level (ave. ploidy level) was determined by flow cytometry as described in Methods. The actual measurements were converted to relative measurements (fraction of wt, third column) using the wild type (wt) values for normalization. The average nuclear size (ave. nuclear size \pm standard error of the mean) corresponds to data from Figure 4A. The fifth column normalizes these size values to the wild type values.

Additional file 5: Nuclear shape changes in crwn1 and crwn4 mutants. Images of representative DAPI-stained adult leaf cell nuclei from wild type, crwn1 and crwn4 mutants were processed by ImageJ software to determine the circularity index $\left.(4 \pi \cdot \text { Area/(perimeter) })^{2}\right)$, as well as a shape index (perimeter $/ \pi \cdot$ major axis) ${ }^{2}$. Nuclei that deviate from a perfect circle (1.0) show a lower circularity index. The shape index highlights different types of deviations from the round shape. Nuclei in the crwn 1 sample show a shape index close to 1.0, indicating consistently round nuclei. The reduced (relative to 1.0) shape indices in the wild-type sample across all nuclear sizes indicate uniformly elongated nuclear shapes. The elevated shape indices characteristic of larger crwn4 nuclei result from the presence of thin projections from the surface of otherwise round nuclei.

Additional file 6: Leaf nuclear preparation and confocal imaging reveals a consistent nuclear thickness across a range of nuclear sizes. Mature leaves were harvested from five individuals in a F2 population segregating both crwn 1 and $c r w n 2$ mutations (F2 plants of a crwn 1 crwn $2 \times$ wild type cross). Consequently, the sample captured a range of nuclear shapes and sizes. The nuclei were fixed, isolated, and prepared for imaging as described for Figure 4. Following DAPI staining, the three-dimensional signal of different nuclei were recorded and reconstructed using a Leica SP5 confocal microscope. The area of each nucleus was measured using ImageJ, while the thickness of each nucleus was determined by the number and thickness of steps on the z-axis necessary to move from the top to the bottom of each nucleus. The different colored dots on the graph correspond to different slides imaged in this experiment. The results indicate that our preparation and imaging procedure generates nuclei with a relatively uniform thickness, mostly in the 2-3 micrometer range, regardless of the size and shape of the nuclei. Further, this thickness is consistent across individual slides.

Additional file 7: Chromocenter changes in crwn double mutants. Nuclei were harvested from developmentally matched rosette leaves from approximately one month-old plants, stained with DAPI, and imaged using epifluorescence microscopy. The area and chromocenter number of randomly-selected individual nuclei $(n=41-52)$ were determined for each genotype, and chromocenter number was plotted against nuclear area. A linear regression line showing the relationship between chromocenter number and nuclear size (as a proxy for endopolyploidy level) was plotted for each genotype.

Additional file 8: Movies S1-3. Three-dimensional reconstruction of nuclei imaged in the fluorescence in situ hybridization experiment. Representative nuclei from wild type (Movie S1), crwn1 crwn2 (Movie S2) and crwn4 (Movie S3) leaf cells were processed by FISH as described in Figure 7. Blue: DAPI, pink: 180-bp centromere repeats, green: 5S RNA genes. The 180-bp centromere repeat and the 5S RNA gene signals were localized to the nuclear periphery in all genotypes.

\section{Competing interests}

The authors declare that they have no competing interests.

\section{Authors' contributions}

HW planned and performed the experiments, prepared the figures, and contributed to the preparation of the manuscript. TAD provided technical advice, generated genetic reagents and contributed to the preparation of the manuscript. EJR supervised the project and wrote the manuscript. All authors read and approved the final manuscript.

\section{Acknowledgements}

We are grateful for the guidance and facilities provided by Wojtek Pawlowski, Choon-lin Tiang, Mamta Srivastava and Boyce Thompson Institute's Plant Cell Imaging Center. We thank Natalie Henkhaus, Erika Hughes, and Molly Shook for helpful comments and discussion. The T-DNA mutant lines used in this study were obtained from the Arabidopsis Biological Resource Center at The Ohio State University. This work was supported by a grant from the National Science Foundation to EJR (MCB-0956820) and from funds provided by the Boyce Thompson Institute.

\section{Author details}

${ }^{1}$ Boyce Thompson Institute for Plant Research, 533 Tower Road, Ithaca, NY 14853, USA. ${ }^{2}$ National Cancer Institute, National Institutes of Health, Bethesda, MD 20892, USA

Received: 3 July 2013 Accepted: 26 November 2013

Published: 5 December 2013

\section{References}

1. Meier I: The plant nuclear envelope. Cell Mol Life Sci 2001, 58(12-13):1774-1780.

2. Gruenbaum Y, Goldman RD, Meyuhas R, Mills E, Margalit A, Fridkin A, Dayani $Y$, Prokocimer $M$, Enosh $A$ : The nuclear lamina and its functions in the nucleus. Int Rev Cytol 2003, 226:1-62.

3. Graumann K, Evans DE: The plant nuclear envelope in focus. Biochem Soc Trans 2010, 38(Pt 1):307-311.

4. Rose A: Open mitosis. In Nuclear envelope dynamics. Edited by Verma DPS, Hong Z. Berlin Heidelberg: Springer; 2007.

5. Dittmer TA, Stacey NJ, Sugimoto-Shirasu K, Richards EJ: Little nuclei genes affecting nuclear morphology in Arabidopsis thaliana. Plant Cell 2007, 19(9):2793-2803.

6. Dittmer TA, Richards EJ: Role of LINC proteins in plant nuclear morphology. Plant Signal Behav 2008, 3(7):485-487.

7. Sakamoto Y, Takagi S: LITTLE NUCLEI 1 and 4 regulate nuclear morphology in Arabidopsis thaliana. Plant Cell Physiol 2013, 54(4):622-633.

8. Masuda K, Xu ZJ, Takahashi S, Ito A, Ono M, Nomura K, Inoue M: Peripheral framework of carrot cell nucleus contains a novel protein predicted to exhibit a long alpha-helical domain. Exp Cell Res 1997, 232(1):173-181.

9. Ciska M, Masuda K, Moreno Diaz de la Espina S: Lamin-like analogues in plants: the characterization of NMCP1 in Allium cepa. J Exp Bot 2013, 64(6):1553-1564.

10. Kimura Y, Kuroda C, Masuda K: Differential nuclear envelope assembly at the end of mitosis in suspension-cultured Apium graveolens cells. Chromosoma 2010, 119(2):195-204.

11. Gardiner J, Overall R, Marc J: Putative Arabidopsis homologues of metazoan coiled-coil cytoskeletal proteins. Cell Biol Int 2011 35(8):767-774.

12. Razafsky D, Hodzic D: Bringing KASH under the SUN: the many faces of nucleo-cytoskeletal connections. J Cell Biol 2009, 186(4):461-472.

13. Sosa BA, Rothballer A, Kutay U, Schwartz TU: LINC complexes form by binding of three KASH peptides to domain interfaces of trimeric SUN proteins. Cell 2012, 149(5):1035-1047.

14. Tapley EC, Starr DA: Connecting the nucleus to the cytoskeleton by SUN-KASH bridges across the nuclear envelope. Curr Opin Cell Biol 2013, 25(1):57-62

15. Zhou X, Graumann K, Evans DE, Meier I: Novel plant SUN-KASH bridges are involved in RanGAP anchoring and nuclear shape determination. J Cell Biol 2012, 196(2):203-211.

16. Graumann K, Evans DE: Plant SUN domain proteins: components of putative plant LINC complexes? Plant Signal Behav 2010, 5(2):154-156.

17. Alonso JM, Stepanova AN, Leisse TJ, Kim CJ, Chen H, Shinn P, Stevenson DK, Zimmerman J, Barajas P, Cheuk R, et al: Genome-wide insertional mutagenesis of Arabidopsis thaliana. Science 2003, 301(5633):653-657.

18. Winter D, Vinegar B, Nahal H, Ammar R, Wilson GV, Provart NJ: An "Electronic Fluorescent Pictograph" browser for exploring and analyzing large-scale biological data sets. PLoS One 2007, 2(8):e718.

19. Chytilova E, Macas J, Sliwinska E, Rafelski SM, Lambert GM, Galbraith DW: Nuclear dynamics in Arabidopsis thaliana. Mol Biol Cell 2000, 11(8):2733-2741.

20. Jovtchev G, Schubert V, Meister A, Barow M, Schubert I: Nuclear DNA content and nuclear and cell volume are positively correlated in angiosperms. Cytogenet Genome Res 2006, 114(1):77-82.

21. Melaragno JE, Mehrotra B, Coleman AW: Relationship between endopolyploidy and cell size in epidermal tissue of Arabidopsis. Plant Cell 1993, 5(11):1661-1668

22. Fransz P, Soppe W, Schubert I: Heterochromatin in interphase nuclei of Arabidopsis thaliana. Chromosome Res 2003, 11(3):227-240. 
23. Fransz P, De Jong JH, Lysak M, Castiglione MR, Schubert I: Interphase chromosomes in Arabidopsis are organized as well defined chromocenters from which euchromatin loops emanate. Proc Natl Acad Sci U S A 2002, 99(22):14584-14589.

24. Schubert V, Berr A, Meister A: Interphase chromatin organisation in Arabidopsis nuclei: constraints versus randomness. Chromosoma 2012, 121(4):369-387.

25. Schubert V, Klatte M, Pecinka A, Meister A, Jasencakova Z, Schubert I: Sister chromatids are often incompletely aligned in meristematic and endopolyploid interphase nuclei of Arabidopsis thaliana. Genetics 2006, 172(1):467-475.

26. Fang $Y$, Spector DL: Centromere positioning and dynamics in living Arabidopsis plants. Mol Biol Cell 2005, 16(12):5710-5718.

27. Bradley MV: Cell and nuclear size in relation to polysomaty and the nuclear cycle. Am J Bot 1954, 41(5):398-402.

28. Burke B: The nuclear envelope: filling in gaps. Nat Cell Biol 2001, 3(12):E273-E274.

29. Levy DL, Heald R: Nuclear size is regulated by importin alpha and Ntf2 in Xenopus. Cell 2010, 143(2):288-298.

30. van Zanten M, Koini MA, Geyer R, Liu Y, Brambilla V, Bartels D, Koornneef M, Fransz P, Soppe WJ: Seed maturation in Arabidopsis thaliana is characterized by nuclear size reduction and increased chromatin condensation. Proc Natl Acad Sci U S A 2011, 108(50):20219-20224.

31. Sugimoto-Shirasu K, Roberts K: "Big it up": endoreduplication and cell-size control in plants. Curr Opin Plant Biol 2003, 6(6):544-553.

32. Epstein HF, Casey DL, Ortiz I: Myosin and paramyosin of Caenorhabditis elegans embryos assemble into nascent structures distinct from thick filaments and multi-filament assemblages. J Cell Biol 1993, 122(4):845-858.

33. Dechat T, Pfleghaar K, Sengupta K, Shimi T, Shumaker DK, Solimando L, Goldman RD: Nuclear lamins: major factors in the structural organization and function of the nucleus and chromatin. Genes Dev 2008, 22(7):832-853.

34. Friedl $P$, Wolf $K$, Lammerding J: Nuclear mechanics during cell migration. Curr Opin Cell Biol 2011, 23(1):55-64.

35. Tamura K, Fukao Y, Iwamoto M, Haraguchi T, Hara-Nishimura I: Identification and characterization of nuclear pore complex components in Arabidopsis thaliana. Plant Cell 2010, 22(12):4084-4097.

36. Tamura K, Hara-Nishimura I: Involvement of the nuclear pore complex in morphology of the plant nucleus. Nucleus 2011, 2(3):168-172.

37. Arumuganathan K, Earle ED: Estimation of nuclear DNA content of plants by flow cytometry. Plant Mol Biol Report 1991, 9:229-233.

38. Golubovskaya IN, Harper LC, Pawlowski WP, Schichnes D, Cande WZ: The pam1 gene is required for meiotic bouquet formation and efficient homologous synapsis in maize (Zea mays L). Genetics 2002, 162(4):1979-1993.

doi:10.1186/1471-2229-13-200

Cite this article as: Wang et al: Arabidopsis CROWDED NUCLEI (CRWN) proteins are required for nuclear size control and heterochromatin organization. BMC Plant Biology 2013 13:200.

\section{Submit your next manuscript to BioMed Central and take full advantage of:}

- Convenient online submission

- Thorough peer review

- No space constraints or color figure charges

- Immediate publication on acceptance

- Inclusion in PubMed, CAS, Scopus and Google Scholar

- Research which is freely available for redistribution

Submit your manuscript at www.biomedcentral.com/submit
( Biomed Central 